\title{
PROGRESSIVE INDUCTOR MODELING VIA A FINITE ELEMENT SUBPROBLEM METHOD
}

\author{
Patrick Dular $^{1,2}$, Laurent Krähenbühl ${ }^{3}$ and Christophe Geuzaine ${ }^{1}$ \\ ${ }^{1}$ University of Liège, Dept. of Electrical Engineering and Computer Science, ACE, B-4000 Liège, Belgium \\ ${ }^{2}$ F.R.S.-FNRS, Fonds de la Recherche Scientifique, Belgium \\ ${ }^{3}$ Université de Lyon, Ampère (CNRS UMR5005), École Centrale de Lyon, F-69134 Écully Cedex, France
}

\begin{abstract}
The modeling of inductors is split into a sequence of progressive finite element subproblems. The source fields generated by the coil conductors alone, with a wire representation, are calculated at first via either the Biot-Savart law or finite elements. The associated reaction fields for each added or modified region, mainly the magnetic cores, and in return for the source conductor regions themselves when massive, are then calculated with finite element models. Changes of magnetic regions go from perfect magnetic properties up to volume linear and nonlinear properties. The resulting subproblem method allows efficient solving of parameterized analyses thanks to a proper mesh for each subproblem and the reuse of previous solutions to be locally corrected.
\end{abstract}

\section{INTRODUCTION}

Instead of solving a complete inductor problem, including coil conductors and magnetic cores, it is here proposed to perform successive finite element (FE) calculations via a subproblem (SP) method (SPM) [1]-[3], mainly by separating the material regions, and giving them models of different accuracy levels. The aim is to lighten the computational efforts in the preliminary stages of a design, e.g. in parameterized analyses, before refining the models. The idea of source and reaction fields is considered but, at the difference with the common method that adds these fields in the whole domain to define the total field, the source fields are here to be defined, via projections, only in the FE mesh of the added regions as local volume sources (VSs) [1]-[3]. They can even be initially reduced to the boundary of the added region, as local surface sources (SSs), which is an important aspect developed here, in particular for efficient calculations with source fields calculated via the Biot-Savart law.

Progressive SPs can tackle the added magnetic core regions at different levels of precision, considering them as perfect magnetic regions, with the source fields acting as SSs, up to linear and nonlinear volume regions. The accuracy of the coil conductor models can increase as well, starting with the wire (filament; with negligible section) representation of the conductors with Biot-Savart models up to their volume FE models, both in magnetostatics and magnetodynamics. Sequences of such SP solutions and/or corrections are developed for the magnetic vector potential $\mathrm{FE}$ magnetodynamic formulation in 2-D and 3-D. They will be illustrated and validated on application examples.

\section{SEQUENCE OF SUBPROBLEMS}

A canonical magnetodynamic problem $p$, to be solved at step $p$ of the SPM, is defined in a domain $\Omega_{p}$, with boundary $\partial \Omega_{p}=\Gamma_{p}=\Gamma_{h, p} \cup \Gamma_{b, p}$. The eddy current conducting part of $\Omega_{p}$ is denoted $\Omega_{c, p}$ and the non-conducting one $\Omega_{c, p}{ }^{C}$, with $\Omega_{p}=\Omega_{c, p} \cup \Omega_{c, p} C$. Stranded conductors belong to $\Omega_{\mathrm{s}, p} \subset \Omega_{c, p}{ }^{C}$. Magnetic field $\boldsymbol{h}_{p}$ and electric current density $\boldsymbol{j}_{p}$ are related to magnetic flux density $\boldsymbol{b}_{p}$ and electric field $\boldsymbol{e}_{p}$, respectively, through the material relations

$$
\boldsymbol{h}_{p}=\mu_{p}^{-1} \boldsymbol{b}_{p}+\boldsymbol{h}_{s, \mathrm{p}}, \boldsymbol{j}_{p}=\sigma_{p} \boldsymbol{e}_{p}+\boldsymbol{j}_{s, \mathrm{p}},
$$

where $\mu_{p}$ is the magnetic permeability (possibly function of $\boldsymbol{b}_{p}$ in a nonlinear material), $\sigma_{p}$ is the electric conductivity, and $\boldsymbol{h}_{\mathrm{s}, \mathrm{p}}$ and $\boldsymbol{j}_{\mathrm{s}, \mathrm{p}}$ are VSs [2], [3]. They can be remnant fields in magnets or fixed current densities in conductors. They can also be defined by

$$
\boldsymbol{h}_{s, p}=\left(\mu_{p}{ }^{-1}-\mu_{q}{ }^{-1}\right) \boldsymbol{b}_{q}, \boldsymbol{j}_{s, p}=\left(\sigma_{p}-\sigma_{q}\right) \boldsymbol{e}_{q},
$$

for changes from $\mu_{q}$ and $\sigma_{q}$ for previous SP $q$ to $\mu_{p}$ and $\sigma_{p}$ for SP $p$ in some regions [2], [3]. Also, BCs are defined for SSs, possibly expressed from previous solutions, i.e.

$\boldsymbol{n} \times\left.\boldsymbol{h}_{p}\right|_{\Gamma_{h, p}}=\boldsymbol{j}_{f, p},\left.\boldsymbol{n} \cdot \boldsymbol{b}_{p}\right|_{\Gamma_{b, p}}=\boldsymbol{f}_{f, p}, \boldsymbol{n} \times\left.\boldsymbol{e}_{p}\right|_{\Gamma_{e, p}} \subset \Gamma_{b, p}=\boldsymbol{k}_{f, p}, \quad(3 \mathrm{a}-\mathrm{b}-\mathrm{c})$ with $\boldsymbol{n}$ the unit normal exterior to $\Omega_{p}$. Some paired portions of $\Gamma_{p}$ can define double layers, with the thin region in between exterior to $\Omega_{p}$; in particular, these will be associated with the boundary of the volume conductors or cores. They are denoted $\gamma_{p}{ }^{+}$and $\gamma_{p}{ }^{-}$and are geometrically defined as a single surface $\gamma_{p}$ with interface conditions (ICs), fixing the discontinuities $\left([\cdot]_{\gamma_{p}}=\left.\left.\cdot\right|_{\gamma_{p}}{ }^{+-} \cdot\right|_{\gamma_{p}}{ }^{-}\right)$

$\left[\boldsymbol{n} \times \boldsymbol{h}_{p}\right]_{\gamma_{p}}=\left[\boldsymbol{j}_{f, p}\right]_{\gamma_{p}},\left[\boldsymbol{n} \cdot \boldsymbol{b}_{p}\right]_{\gamma_{p}}=\left[\boldsymbol{f}_{f, p}\right]_{\gamma_{p}},\left[\boldsymbol{n} \times \boldsymbol{e}_{p}\right]_{\gamma_{p}}=\left[\boldsymbol{k}_{f, p}\right]_{\gamma_{p}} .(4 \mathrm{a}-\mathrm{b}-\mathrm{c})$

With the magnetic vector potential $\boldsymbol{a}_{p}$ and electric scalar potential $v_{p}$ defined via $\boldsymbol{b}_{p}=\operatorname{curl} \boldsymbol{a}_{p}$ and $\boldsymbol{e}_{p}=-\partial_{t} \boldsymbol{a}_{p}-\operatorname{grad} v_{p}=$ $-\partial_{t} \boldsymbol{a}_{p}-\boldsymbol{u}_{p}$, and the resulting $\mathrm{BC}$ and IC

$$
\boldsymbol{n} \times\left.\boldsymbol{a}_{p}\right|_{\Gamma_{b, p}}=\boldsymbol{a}_{f, p}, \quad\left[\boldsymbol{n} \times \boldsymbol{a}_{p}\right]_{\gamma_{p}}=\left[\boldsymbol{a}_{f, p}\right]_{\gamma_{p}},
$$

the $\boldsymbol{a}_{p}$ weak formulation of the magnetodynamic problem is obtained from the weak form of the Ampère equation, i.e. [2]

$$
\begin{aligned}
& \left(\mu_{p}^{-1} \operatorname{curl} \boldsymbol{a}_{p}, \operatorname{curl} \boldsymbol{a}^{\prime}\right)_{\Omega_{p}}+\left(\boldsymbol{h}_{s, p}, \operatorname{curl} \boldsymbol{a}^{\prime}\right)_{\Omega_{p}}-\left(\boldsymbol{j}_{s, p}, \boldsymbol{a}^{\prime}\right)_{\Omega_{p}} \\
& +\left(\sigma_{p} \partial_{t} \boldsymbol{a}_{p}, \boldsymbol{a}^{\prime}\right)_{\Omega_{c, p}}+\left(\sigma_{p} \boldsymbol{u}_{p}, \boldsymbol{a}^{\prime}\right)_{\Omega_{c, p}}+<\boldsymbol{n} \times \boldsymbol{h}_{p}, \boldsymbol{a}^{\prime}>_{\Gamma_{h, p} \gamma_{p}} \\
& +<\left[\boldsymbol{n} \times \boldsymbol{h}_{p}\right]_{\gamma_{p}}, \boldsymbol{a}^{\prime}>_{\gamma_{p}}=0, \forall \boldsymbol{a}^{\prime} \in F_{p}^{1}\left(\Omega_{p}\right),
\end{aligned}
$$

where $F_{p}{ }^{1}\left(\Omega_{p}\right)$ is a curl-conform function space defined on $\Omega_{p}$, gauged in $\Omega_{c, p}{ }^{C}$, and containing the basis functions for $\boldsymbol{a}_{p}$ and for the test function $\boldsymbol{a}^{\prime}$ (at the discrete level, this space is defined by edge FEs; the gauge is based on the tree-co-tree technique); $(\cdot, \cdot)_{\Omega}$ and $<\cdot, \cdot>_{\Gamma}$ denote a volume integral in $\Omega$ and a surface integral on $\Gamma$, respectively, of the product of their field arguments.

With the SPM, a complete problem is split into a series of SPs that define a sequence of changes, with the complete solution given by the sum of the SP solutions [1]-[3]. Each SP is defined in its particular domain and mesh, usually overlapping those of the other SPs. At the discrete level, this allow distinct meshes with suitable refinements and possible domain overlapping between separate SPs. 


\section{PROGRESSIVE INDUCTOR PROBLEMS}

\section{A. Conductor and core models}

Coil conductor alone (COIL-BS) - A volume conductor, possibly stranded, can be first simplified to a wire geometry $\Omega_{s, p}$ alone, from which the generated field is defined via the Biot-Savart formula, being a direct solution (no need of FE calculation) of the related SP $p \equiv$ coil-BS, with fixed source $\boldsymbol{j}_{\mathrm{s}, \mathrm{p}}$ in $\Omega_{\mathrm{s}, p}$. Fields $\boldsymbol{b}_{p}$ and $\boldsymbol{a}_{p}$, with $\boldsymbol{e}_{p}=-\partial_{t} \boldsymbol{a}_{p}$, are then defined via line integrals along the wire. They are to be calculated afterward only in some particular regions, as VSs or SSs when adding other regions or for a change to a volume conductor.

Perfect magnetic core (CORE-PMC) - An SP $p \equiv$ corepmc is defined in a new domain $\Omega_{p}$ by considering some added core regions $\Omega_{p, i}$ ( $i$ is the region index) as being perfect, i.e. of infinite $\mu_{p}$. The interior of $\Omega_{p, i}$, with zero total $\boldsymbol{h}$ inside, is extracted from the studied domain $\Omega_{p}$ and treated via BC (3a) to fix a zero trace of total $\boldsymbol{h}$ on their boundaries $\Gamma_{p, i}=\partial \Omega_{p, i}$, thus coupling both the unknown field and the field from previous $\mathrm{SP}(\mathrm{s}) q$, acting as a SS [3], i.e.

$$
\boldsymbol{n} \times\left.\boldsymbol{h}_{p}\right|_{p, i}=-\boldsymbol{n} \times \boldsymbol{h}_{q} \mid \Gamma_{p, i} .
$$

The solution can serve as a reference solution for any finite permeability further considered.

Volume core (CORE-VOL) - A volume magnetic core $\Omega_{p, i}$ is considered in an SP $p \equiv$ core-vol. For a newly added core, VSs (2a-b) using previous solution(s) $q$ are used. If the core has been initially added as a perfect magnetic core, considering also a zero solution $\boldsymbol{b}_{q}$ in $\Omega_{p, i}$, the VSs there are zero; $\boldsymbol{b}_{q}$ is considered to be carried in the double layer of $\Gamma_{p, i}$. A trace discontinuity of $\boldsymbol{a}_{q}\left(\boldsymbol{b}_{q}\right)$ thus occurs, of which the opposite value defines an SS for SP $p$ in $(5 b)((4 b))$, strongly expressed in function space $F_{p}{ }^{1}(\Omega)$, i.e., also with a zero SS in $(4 a)$,

$$
\left[\boldsymbol{n} \times \boldsymbol{a}_{p}\right]_{\Gamma_{p, i}}=-\boldsymbol{n} \times \boldsymbol{a}_{q} \mid \Gamma_{p, i}, \quad\left[\boldsymbol{n} \times \boldsymbol{h}_{p}\right]_{\Gamma_{p, i}}=0 .
$$

Changes to nonlinear magnetic properties can be done with VS (2a), with the new $\mu_{p}{ }^{-1}$ function of the total field $\left(\boldsymbol{b}_{q}+\boldsymbol{b}_{p}\right)$, i.e., $\boldsymbol{\mu}_{p}^{-1}=\boldsymbol{\mu}_{p}{ }^{-1}\left(\boldsymbol{b}_{q}+\boldsymbol{b}_{p}\right)$. Nonlinear iterations are needed for the related SP up to the convergence of $\boldsymbol{a}_{p}$. Other changes of the core model could concern changes to equivalent properties of the core laminations in a homogenized model or could go up to the fine description of the laminations.

Volume coil (COIL-VOL) - A wire conductor $\Omega_{s, q}$ from a previous SP $q$ can be corrected to its actual volume geometry $\Omega_{s, p}\left(\Omega_{s, q} \subset \Omega_{s, p}\right)$ by a new SP $p \equiv$ coil-vol, carrying an unchanged total current and defined, with its surrounding, with a FE mesh. To overcome the singularity proper to the Biot-Savart field along wire $\Omega_{s, q}$, the key is to get rid of the singular solution $q$ inside $\Omega_{s, p}$, keeping the solution unchanged outside, simultaneously to adding the volume conductor $\Omega_{s, p}$. As it will be explained, this is done via ICs with SSs through $\Gamma_{s, p}=\partial \Omega_{s, p}$, i.e.

$$
\left[\boldsymbol{n} \times \boldsymbol{h}_{p}\right]_{\Gamma_{s, p}}=-\boldsymbol{n} \times\left.\boldsymbol{h}_{q}\right|_{\Gamma_{s, p}},\left[\boldsymbol{n} \cdot \boldsymbol{b}_{p}\right]_{\Gamma_{s, p}}=-\left.\boldsymbol{n} \cdot \boldsymbol{b}_{q}\right|_{\Gamma_{s, p}},
$$

together with $\boldsymbol{j}_{s, p}$ fixed as a VS in $\Omega_{s, p}$ (static), or the total current fixed in $\Omega_{s, p}$ (dynamic). Solution $q$ includes the possible added core solution. The Biot-Savart calculation is required only on $\Gamma_{s, p}$ to evaluate the SSs. SS (9a) is weakly expressed in the weak formulation (6) whereas (9b) strongly fixes a discontinuity of $\boldsymbol{n} \times \boldsymbol{a}_{p}$ through $\Gamma_{s, p}$ in $F_{p}{ }^{1}\left(\Omega_{p}\right)$.

\section{B. SP Corrections and their sources}

Any SP $p$ is defined as a correction of a previous (or several) $\mathrm{SP}(\mathrm{s}) q$, without involving the already considered sources. It requires VSs and/or SSs in some regions $\Omega_{p, i}$ or $\Gamma_{p, i}$ evaluated from previous $\mathrm{SP}(\mathrm{s}) q$. These sources, coming from previous meshes or Biot-Savart evaluations of SPs $q$, have to be properly discretized in the mesh of SP $p$ to assure the conformity of the sequenced FE weak formulations. They are obtained by means of Galerkin projections of the primary field $\boldsymbol{a}_{q}$ between the meshes [3]. For a VS, an alternative to the projection on a volume $\Omega_{p, i}$ consists in evaluating and projecting the source on its surface $\Gamma_{p, i}$, that then defines the $\mathrm{BC}$ of a physical local FE problem. A Biot-Savart VS gains at being processed in this way. A change with a significant effect on the previously solved SPs has to be further considered as a source for these, which thus requires iterative corrections. Various correction schemes will be studied (e.g., Fig. 1, using SSs).

\section{Inductance and resistance calculation}

The self inductance of a wire inductor, and the possible mutual inductances with other wire conductors, can be calculated via double integral Neumann formulas. The resistance can be approximated as well. After a volume correction SP $p$, the corrected inductance can be shown to be advantageously obtained with the solution $\boldsymbol{a}_{p}$ only in $\Omega_{s, p}$, i.e. via $\left(\boldsymbol{j}_{s, p}, \boldsymbol{a}_{p}\right)_{\Omega_{s, p}}$ defining the total magnetic flux, thus as the new global value without any reference to the wire inductance approximation. An added magnetic core in an SP gives an inductance change that can be calculated by a volume integral limited to the added region, which is another advantage of the SPM.

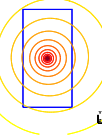

(a)

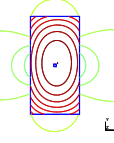

(b)

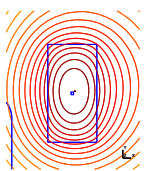

(c)

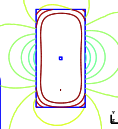

(d)

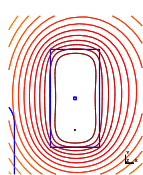

(e)
Fig. 1. Field lines in the surrounding of a conductor (portion of a full geometry, wire position shown): (a) wire conductor Biot-Savart field $\left(\boldsymbol{b}_{q}\right),(\mathrm{b}-$ c) static volume correction $\left(\boldsymbol{b}_{p, s t a}\right)$ and total field, (d-e) dynamic volume correction field $\left(\boldsymbol{b}_{p, d y n}\right)$ and total field. The volume correction gives the total field in the volume conductor, is discontinuous through the inductor boundary and quickly decreases outside.

\section{CONCLUSION}

The developed FE-SPM allows to split inductor modeling into SPs of lower complexity regarding meshing operations and computational aspects. Source field FE or Biot-Savart calculations are followed by approximate reaction field solutions related to approximate $\mathrm{BCs}$, up to their accurate volume distributions in both coil conductor and magnetic core. Significant corrections are progressively obtained, for the nonlinear magnetic properties and the skin and proximity effects, and the related inductances and resistances.

\section{REFERENCES}

[1] P. Dular, R. V. Sabariego, L. Krähenbühl. "Subdomain perturbation finite element method for skin and proximity effects in inductors", COMPEL, Vol. 27, No. 1, pp. 72-84, (2008)

[2] P. Dular, L. Krähenbühl, R.V. Sabariego, M. V. Ferreira da Luz, P. KuoPeng, C. Geuzaine. "A finite element subproblem method for position change conductor systems", IEEE Trans. Magn., vol. 48, no. 2, pp. $403-$ 406, (2012)

[3] P. Dular, V. Péron, R. Perrussel, L. Krähenbühl, C. Geuzaine. "Perfect conductor and impedance boundary condition corrections via a finite element subproblem method", IEEE Trans. Magn., vol. 50, no. 2, article 7000504 (2014). 